\title{
Correction: Optimality and duality theorems in nonsmooth multiobjective optimization
}

Kwan Deok Bae and Do Sang Kim*

* Correspondence: dskim@pknu.ac. $\mathrm{kr}$

Department of Applied

Mathematics, Pukyong National University, Busan 608-737, Korea
We wish to indicate the following corrections to our original paper [1].

(1) The first sentence in Definition 2.2, we delete " $i \in\{1,2, \cdots, p\}$ ".

(2) The first sentence in Definition 2.2, we replace

$$
f_{i}(x)+s\left(x \mid D_{i}\right) \nless f_{i}\left(x^{0}\right)+s\left(x^{0} \mid D_{i}\right)
$$

to

$$
f(x)+s(x \mid D) \nless f\left(x^{0}\right)+s\left(x^{0} \mid D\right) .
$$

(3) The second sentence in Definition 2.3, we delete " $i \in\{1,2, \cdots, p\}$ ".

(4) The second sentence in Definition 2.3, we replace

$$
f_{i}(x)+s\left(x \mid D_{i}\right) \nless f_{i}\left(x^{0}\right)+s\left(x^{0} \mid D_{i}\right)+c_{i}|| x-x^{0} \|^{m}
$$

to

$$
f(x)+s(x \mid D) \nless f\left(x^{0}\right)+s\left(x^{0} \mid D\right)+c|| x-x^{0} \|^{m} .
$$

(5) The second sentence in Definition 2.4, we delete " $i \in\{1,2, \cdots, p\}$ ".

(6) The second sentence in Definition 2.4, we replace

$$
\prime \prime f_{i}(x)+s\left(x \mid D_{i}\right) \nless f_{i}\left(x^{0}\right)+s\left(x^{0} \mid D_{i}\right)+c_{i}|| x-x^{0} \|^{m}
$$

to

$$
f(x)+s(x \mid D) \nless f\left(x^{0}\right)+s\left(x^{0} \mid D\right)+c\left\|x-x^{0}\right\|^{m} .11 .
$$

(7) The second sentence in the proof of Theorem 2.1, we replace. " $c_{i}>0, i=1, \ldots, p$ " to " $c \in$ intR $R_{+}^{\text {p". }}$

(8) The second sentence in the proof of Theorem 2.1, we replace

$$
f_{i}(x)+s\left(x \mid D_{i}\right) \nless f_{i}\left(x^{0}\right)+s\left(x^{0} \mid D_{i}\right)+c_{i}|| x-x^{0} \|^{m}
$$

to

$$
f(x)+s(x \mid D) \nless f\left(x^{0}\right)+s\left(x^{0} \mid D\right)+c|| x-x^{0} \|^{m} .
$$

(9) In equation (3.8), we replace " $c_{i}$ " to " $d_{i}$ ".

(10) The eighth sentence in the proof of Theorem 3.3, we replace "where $c=a e$," to "where $d=a e^{\prime \prime}$. 
(11) The ninth sentence in the proof of Theorem 3.3, we replace " $c \in$ int $R^{p "}$ to " $d_{i}>0$, $i=1, \cdots, p$, .

(12) The ninth sentence in the proof of Theorem 3.3, we replace " $c_{i}$ " to " $d_{i}$ ".

(13) The tenth sentence in the proof of Theorem 3.3, we replace " $c_{i}$ " to " $d_{i}$ ".

(14) The tenth sentence in the proof of Theorem 3.3, we replace " $c_{i}$ " to " $d_{i}$ ".

(15) In equation (4.8), we replace " $c_{i}$ " to " $d_{i}$ ".

(16) The tenth sentence in the proof of Theorem 4.1, we replace "where $c=a e$," to "where $d=a e^{\text {". }}$.

(17) The eleventh sentence in the proof of Theorem 4.1, we replace " $c \in$ int $R^{p "}$ to " $d_{i}>0, i=1, \cdots, p$, .

(18) The eleventh sentence in the proof of Theorem 4.1, we replace " $c_{i}$ " to " $d_{i}$ ".

(19) The twelfth sentence in the proof of Theorem 4.1, we replace " $c \in$ int $R^{p}$," to " $d_{i}>0, i=1, \cdots, p$, .

(20) The twelfth sentence in the proof of Theorem 4.1 , we replace " $c_{i}$ " to " $d_{i}$ ".

(21) The twelfth sentence in the proof of Theorem 4.1, we replace " $i=1, \cdots, p$." to " $i=1, \cdots, p$, .

(22) The fourth sentence in the proof of Theorem 4.2, we replace " $c_{i}$ " to " $c$ ".

(23) The fourth sentence in the proof of Theorem 4.2, we replace

$$
\begin{aligned}
& f_{i}\left(x^{0}\right)+\left(x^{0}\right)^{T} w_{i}^{0}+c_{i}\left\|u-x^{0}\right\|^{m} \\
& \nless f_{i}(u)+u^{T} w_{i}, i=1, \cdots, p .
\end{aligned}
$$

to

$$
\begin{aligned}
& f\left(x^{0}\right)+\left(x^{0}\right)^{T} w^{0}+c\left\|u-x^{0}\right\|^{m} \\
& \nless f(u)+u^{T} w .
\end{aligned}
$$

1. Bae, Kim, : Optimality and Duality Theorems in Nonsmooth Multiobjective Optimization. Fixed Point Theory and Applications. 2011, 42 (2011). doi:10.1186/1687-1812-2011-42 journal and benefit from:

Convenient online submission

- Rigorous peer review

- Immediate publication on acceptance

- Open access: articles freely available online

- High visibility within the field

- Retaining the copyright to your article 